\title{
Determination of In Vitro Membrane Permeability by Analysis of Intracellular and Extracellular Fluorescein Signals in Renal Cells
}

\author{
ANDREAS NITSCH ${ }^{1,2}$, LYUBOMIR HARALAMBIEV $^{2,3}$, REBEKKA EINENKEL $^{4}$, DAMIÁN O. MUZZIO ${ }^{4}$, \\ MAREK T. ZYGMUNT ${ }^{4}$, AXEL EKKERNKAMP ${ }^{2,3}$, MARTIN BURCHARDT $^{1}$ and MATTHIAS B. STOPE ${ }^{1}$ \\ ${ }^{1}$ Department of Urology, University Medicine Greifswald, Greifswald, Germany \\ ${ }^{2}$ Department of Trauma, Reconstructive Surgery and Rehabilitation Medicine, \\ University Medicine Greifswald, Greifswald, Germany; \\ ${ }^{3}$ Department of Trauma and Orthopaedic Surgery, BG Clinic Trauma Hospital Berlin gGmbH, Berlin, Germany; \\ ${ }^{4}$ Department of Obstetrics and Gynecology, University Medicine Greifswald, Greifswald, Germany
}

\begin{abstract}
Background/Aim: The structural integrity of the eukaryotic cytoplasmic membrane is of crucial importance for its cell biological function and thus for the survival of the cell. Physical and chemical noxae can interact in various ways with components of the cytoplasmic membrane, influence its permeability and thus mediate toxic effects. In the study presented, changes in membrane permeability were quantified by intracellular accumulation of a fluorescent dye and by the release of the fluorescent dye from dye-loaded cells. Materials and Methods: Non-malignant (RC-124) and malignant (786$O$, Caki-1) renal cells were permeabilized with different concentrations of Triton X-100. The permeability of the membrane was determined at the single-cell level by the uptake of the dye into the cell inner by flow cytometry. In addition, a fluorescence plate reader was used to detect and quantify the release of the dye into the cell culture supernatant. Results: Both malignant and non-malignant cells showed a dosedependent alteration of membrane permeability after treatment with Triton $X-100$. In the presence of the fluorescent dye, significantly more dye was introduced into the permeabilized cells compared to control incubations. Vice versa, Triton $X$ 100-treated and dye-loaded cells released significantly more dye into the cell culture supernatant. Conclusion: The
\end{abstract}

This article is freely accessible online.

Correspondence to: Lyubomir Haralambiev, University Medicine Greifswald, Department of Trauma, Reconstructive Surgery and Rehabilitation Medicine, Ferdinand Sauerbruch Straße, 17475 Greifswald, Germany, Tel: +49 38348622541, Fax: +49 3834866013, e-mail: lyubomir.haralambiev@uni-greifswald.de

Key Words: Membrane permeability, fluorescein diacetate, fluorescence. combination of measurement of intracellular accumulated and extracellular released dye can quantifiably detect changes in membrane permeability due to cell-membrane damage. The combination of two different measurement methods offers additional value in reliable detection of membrane-damaging, potentially toxic influences.

Eukaryotic cells are bound by the cytoplasmic membrane that separates intracellular space from extracellular space. In addition, the cytoplasmic membrane plays an important role in the exchange of compounds between cells and their environment. As a semipermeable barrier, the cytoplasmic membrane is responsible for the maintenance of electrochemical and osmotic gradients and thus essential for the physiological functions of the cell (1). The cytoplasmic membrane consists of a double layer of lipids, which enables the passage of lipophilic molecules. In addition, there are a number of highly specific transport systems that mediate and regulate the entry and release of hydrophilic substances (2).

Fluorescein is a hydrophilic fluorescent dye with a broad range of applications in cell biology (3). The derivate fluorescein diacetate (FDA), esterified with two acetate groups, has lipophilic properties and is therefore permeable to membranes. The acetate groups are cleaved within the cell by cellular esterases. As a result, FDA loses its membrane permeability after penetration into the cell, can no longer diffuse out of the cell, and is therefore ideal for fluorescent labelling of living cells (4-6).

In the assays described below, changes in membrane permeability were determined using two different approaches and two different measurement methods. The intracellular enrichment of FDA was determined by flow cytometry. The release of FDA was analyzed in the cell-free supernatant of FDA-loaded cells with a fluorescence plate reader. 


\section{Materials and Methods}

Cell culture. Human renal cell cancer (RCC) cell lines 786-O and Caki-1, as well as the non-malignant renal cell line RC-124 (all Cell Lines Service, Eppelheim, Germany) were propagated in RPMI 1640 medium with $2 \mathrm{mM} \mathrm{L-glutamine,} 1 \%$ penicillin/streptomycin $(\mathrm{P} / \mathrm{S})$ and $10 \%$ fetal bovine serum (786-O), Minimal Essential Medium (MEM) with $79.6 \mathrm{mg} / \mathrm{l}$ non-essential amino acids, $2 \mathrm{mM} \mathrm{L}$-glutamine, $1 \mathrm{mM}$ sodium pyruvate, $1 \% \mathrm{P} / \mathrm{S}$ and $10 \%$ fetal bovine serum, and McCoys 5a medium with $2 \mathrm{mM}$ L-glutamine, $1 \% \mathrm{P} / \mathrm{S}$ and $10 \%$ fetal bovine serum, respectively (all reagents from PAN Biotech, Aidenbach, Germany). All cells were incubated at $37^{\circ} \mathrm{C}$ and $5 \% \mathrm{CO}_{2}$.

For experiments, nearly confluent cells were suspended by trypsin/ethylendiamine tetra-acetic acid (PAN Biotech) treatment and adjusted to 106 cells/ $\mathrm{ml}$ with stop solution [phosphate-buffered saline (PBS) with $10 \%$ fetal bovine serum; PAN Biotech] and stored on ice.

FDA-uptake assay. FDA uptake (Figure 1) was determined by flow cytometry, therefore cells were stained with both FDA and ethidium bromide (EtBr) (7). Cell suspension ( $400 \mu \mathrm{l})$ was mixed with $200 \mu \mathrm{l}$ PBS with Triton X-100 in concentrations from $10-5 \%$ to $10-2 \%$ and $200 \mu \mathrm{l}$ staining solution $(120 \mu \mathrm{g} / \mathrm{ml} \mathrm{EtBr}$ and $20 \mu \mathrm{g} / \mathrm{ml} \mathrm{FDA} \mathrm{in} \mathrm{PBS})$ and incubated on ice for $5 \mathrm{~min}$ in the dark. After addition of $2 \mathrm{ml}$ PBS, cells were sedimented ( $5 \mathrm{~min}, 300 \times g, 4^{\circ} \mathrm{C}$ ), resuspended in $300 \mu \mathrm{l}$ PBS and analyzed in a BD FACSCanto ${ }^{\mathrm{TM}}$ flow cytometer with BD FACSDivaTM 6.0 software (BD Biosciences, Heidelberg, Germany). For analysis of cell debris, doublets and dead cells were excluded by standard gating strategies based on forward- and sidescatter parameters (Figure 2). The mean fluorescence intensity (MFI) was calculated with FlowJo Software Version 10 (Tree Star Inc, Ashland, OR, USA).

FDA-release assay. To determine the FDA release of permeabilized cells, cells were incubated for $30 \mathrm{~min}$ in the dark on ice with staining solution $(5 \mu \mathrm{g} / \mathrm{ml}$ FDA in PBS). Subsequently, cells were washed three times $\left(3 \mathrm{~min}, 150 \times \mathrm{g}, 4^{\circ} \mathrm{C}\right.$ with subsequent resuspension in $500 \mu \mathrm{l}$ PBS) and $200 \mu \mathrm{l}$ of the cell suspension was treated with $10-3 \%$ Triton $\mathrm{X}-100$. Permeabilized cells were then sedimented $\left(3 \mathrm{~min}, 27 \times \mathrm{g}, 4^{\circ} \mathrm{C}\right)$. After an incubation of $20 \mathrm{~min}, 100$ $\mu l$ of the cell-free supernatant was analyzed in an Infinite m200 Pro plate reader (Tecan, Männedorf, Switzerland) at an excitation wavelength of $300 \mathrm{~nm}$ and with an emission wavelength of $520 \mathrm{~nm}$. Data were expressed as relative fluorescence units (RFU).

Data analysis. Microsoft Excel Version 1903 (Microsoft Corp., Redmond, WA, USA) and GraphPad Prism Version 7.04 (GraphPad Software Inc., La Jolla, CA, USA) were used for data analysis and visualization. The statistical analysis of the FDA release assay data was performed by paired $t$-test, the FDA-uptake assay data by ANOVA with Tukey post-hoc test. Results of $p \leq 0.05$ of at least three replicates were considered significant and data are given as the mean \pm SD.

\section{Results}

In order to investigate altered FDA flow rate through the cytoplasmic membrane, the uptake of the dye was determined by flow cytometry. After incubation with FDA and different concentrations of Triton X-100, cells were analyzed by flow cytometry. With increasing concentrations of the surfactant, a dose-dependent decrease of the intracellular FDA signal was observed. This was seen in 786-O cells $(p<0.001)$ and Caki- 1 cells $(p=0.004)$ relatively strongly. RC-124 cells on the other hand showed a less pronounced response to the higher Triton X-100 concentrations $(p=0.148)$ (Figure 3A).

To evaluate the Triton X-100-dependent alterations in membrane permeability, the release of FDA from the cells into the extracellular space was investigated. Cells were incubated with FDA, washed, and then the cell culture supernatant was analyzed in a fluorescence plate reader. Incubation with $10-3 \%$ Triton $\mathrm{X}-100$ resulted in a significantly increased and statistically significant release of FDA from 786-O cells (2.5-fold, $p<0.001)$ and $\mathrm{RC}-124$ cells (7.6-fold, $p=0.005$ ) compared to controls. With Caki-1 cells, however, the effect was distinctly more moderate but still significant (1.1-fold, $p=0.030$ ) (Figure 3B).

\section{Discussion}

Determination of FDA uptake is a fast and cost-effective method for the detection of changes in membrane permeability (5). The fluorescent dye fluorescein is membrane-permeable due to derivatization to FDA and can enter cells without specific uptake mechanisms. In the intracellular space, the hydrolysis of the dye molecule takes place through cellular esterases, whereby the two acetate residues are cleaved off. As a result, (i) the fluorescein molecule loses its membrane permeability and remains localized in the cell, and (ii) the dye becomes excitable and measurable. In the acetate form, the fluorescent dye is inactive and cannot be detected at $550 \mathrm{~nm}$. Free, extracellular FDA therefore does not produce any background signal.

However, these properties are also the reason for the limitation of the use of FDA for the determination of membrane permeability. If noxae which lead to activation and membrane impermeability of FDA through (photo)chemical or nonspecific enzymatic activities are investigated in the FDA-uptake assay, the measurements may be corrupted (8-11). Furthermore, experimental parameters that lead to changes in cell physiology, e.g. the modulation of protein or microRNA expression, might also influence the metabolic activity of the cell. If FDA-transforming esterases are also affected, altered enzymatic activation of the dye molecules would simulate changes in membrane permeability $(12,13)$. These limitations can be partially compensated for by the FDA-release assay. Cells are loaded with FDA and only then treated. A direct influence of the treatment on the dye itself can therefore be excluded. However, this only applies to the direct interaction between the physical/chemical treatment and the FDA molecules. If the treatment triggers changes in the cellular metabolism, or if genetic manipulations are carried out directly in the cell, 


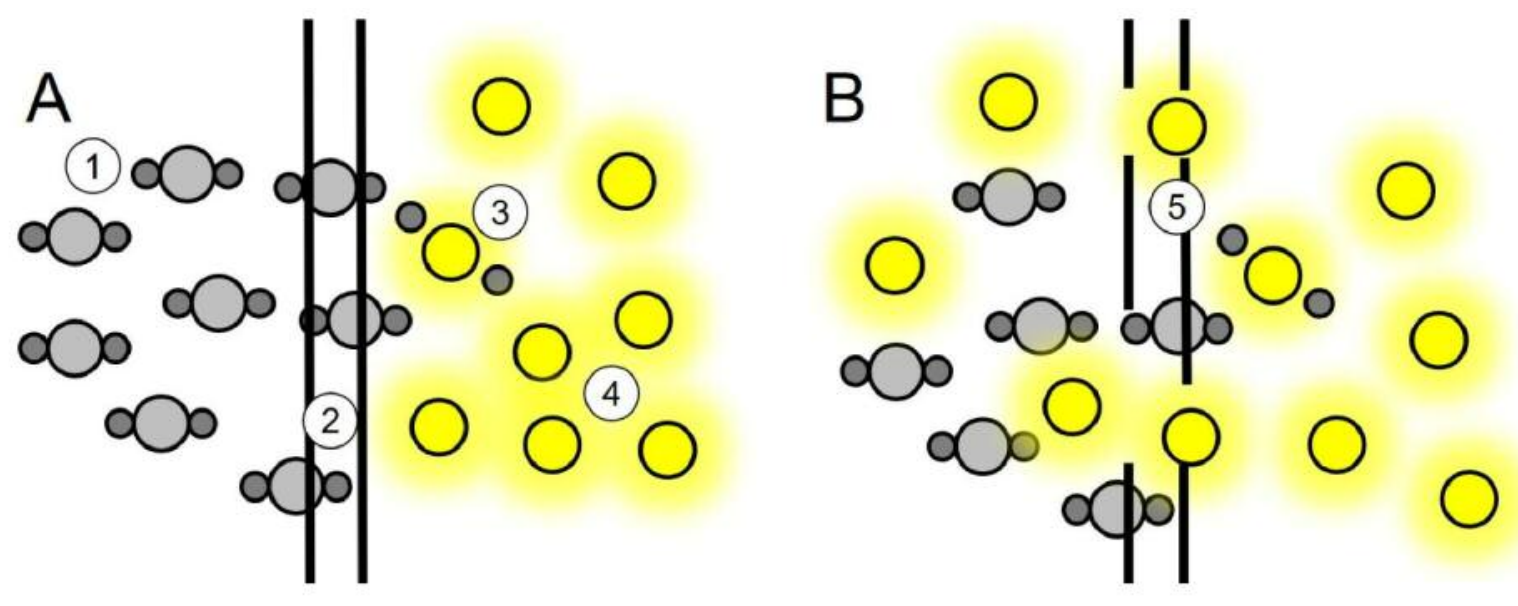

Figure 1. Measurement and mode of action of fluorescein diacetate (FDA) uptake and release assay. A: FDA itself does not fluoresce (1). Due to its membrane permeability, FDA can diffuse into the cell interior (2) where it is metabolized by cellular esterases (3) and transformed into the fluorescent dye fluorescein (4). B: When membrane permeability is altered, FDA molecules, as well as activated fluorescein molecules, can increasingly pass through the membrane (5). This enables the measurement of altered extracellular (FDA-release assay) as well as intracellular (FDA-uptake assay) fluorescence signals.

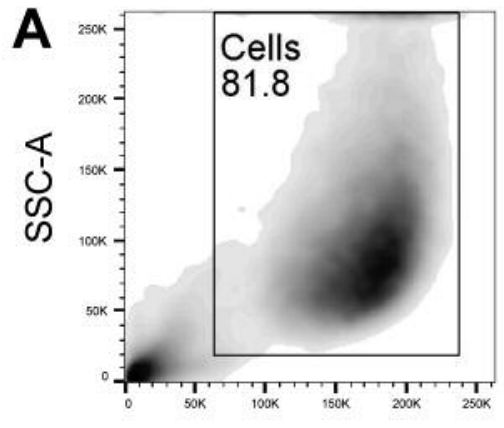

FSC-H

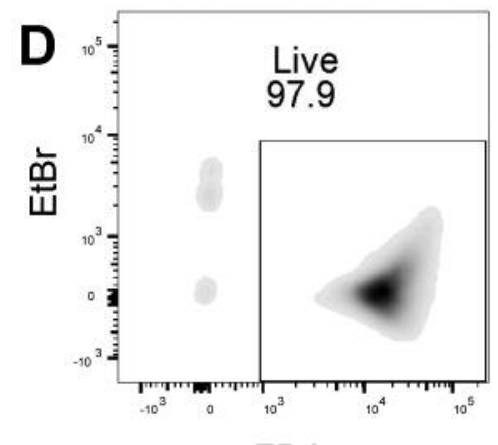

FDA
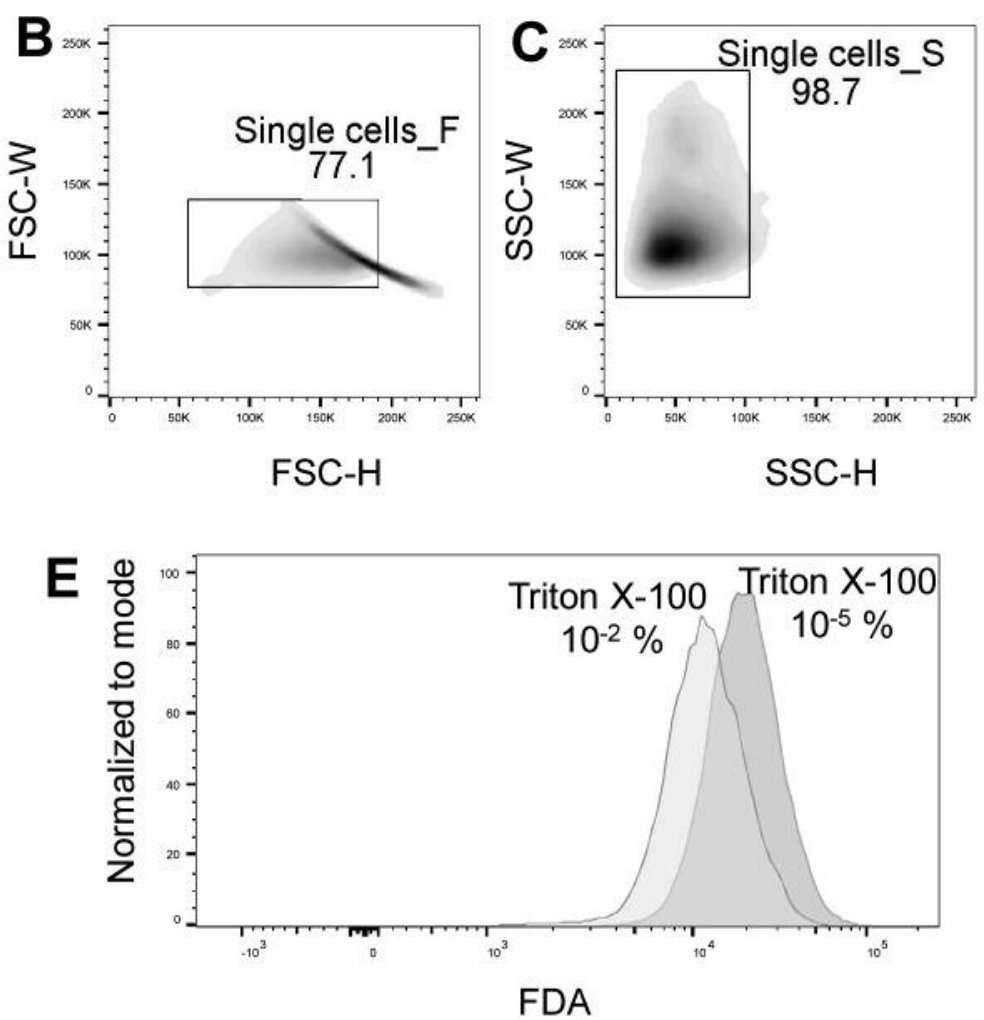

Figure 2. Fluorescein diacetate (FDA) content per cell was analyzed by flow cytometry. Debris (A) and doublets (B, C) were excluded by forward and side-scatter characteristics. Living cells were defined as ethidium bromide (EtBr)-negative and FDA-positive events (D). For analysis of data, the mean fluorescence intensity (MFI) of FDA was compared (E). SSC-A: side-scatter area, SSC-H: side-scatter height, FSC-W: forward-scatter width, FSC-H: forward-scatter height. 
786-0
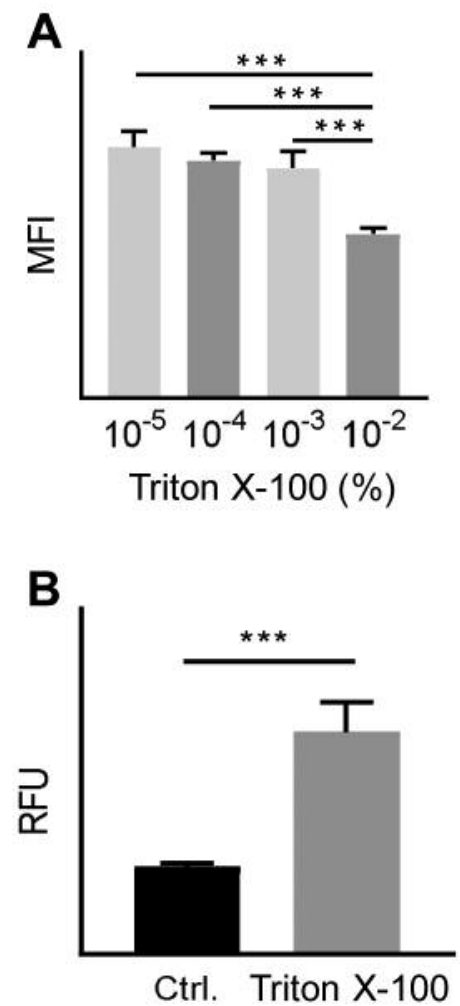

Caki-1
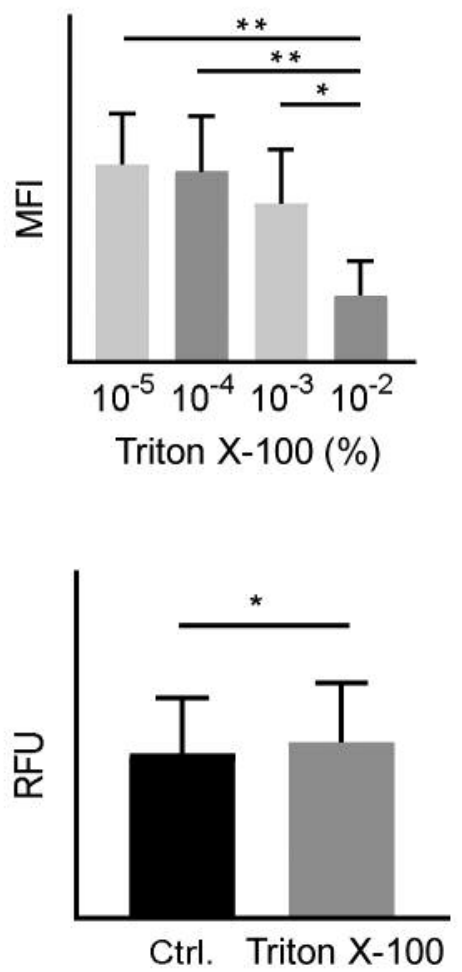

RC-124
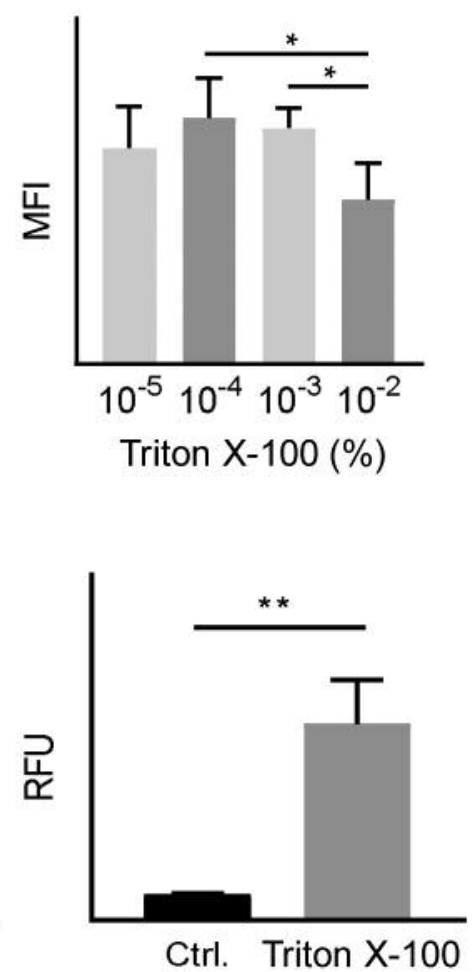

Figure 3. Determination of membrane permeability alterations by fluorescein diacetate (FDA) uptake (A) and release (B) assays. In the FDA-uptake assay, FDA- and ethidium bromide (EtBr)-stained malignant 786-O and Caki-1 cells, and non-malignant RC-124 cells were incubated with Triton $X-100$ at the indicated concentrations. Afterwards, the flow cytometric analysis of living single cells was performed. For the FDA-release assay, 786-O, Caki-1, and RC-124 cells were incubated with FDA, washed, and incubated in FDA-free phosphate-buffered saline (PBS). After an incubation time of $20 \mathrm{~min}$ the cell-free cell culture supernatant was analyzed in a fluorescence plate reader (excitation: $300 \mathrm{~nm}$; emission $520 \mathrm{~nm}$ ). Data are the mean $\pm S D$. Significantly different at $* p \leq 0.05, * * \leq \leq 0.01$ and $* * * p \leq 0.001$ according to ANOVA (A) and t-test (B). MFI: Mean fluorescence intensity, $R F U$ : relative fluorescence units.

this can nevertheless lead to altered metabolism of intracellular FDA molecules.

A further misrepresentation of the measurements can occur if cells are mechanically destroyed and lysed (14). FDA liberation in the FDA-release assay in such a case would erroneously imply an increased permeability of the cytoplasmic membrane. This in turn can be ruled out by analysis with the FDA-uptake assay. In the flow cytometric evaluation of the cells, only living intact cells are included in the measurements.

In this study, RCC cells (786-O, Caki-1) and nonmalignant renal cells (RC-124) were investigated. All three cell lines showed slightly different effects after treatment with Triton X-100 in the FDA-uptake assay as well as the FDA-release assay. As already determined in other studies $(15,16)$, the membrane-permeabilizing effect of Triton $\mathrm{X}-100$ was not different between malignant and non- malignant renal cells. It can therefore be assumed that both the FDA-uptake test and the FDA-release test can be performed with cancer cells as well as with normal cells. It is therefore highly probable that the technique can also be applied to cells of other origins.

In summary, the combination of FDA-uptake assay and FDA-release assay can overcome many limitations of singleuse FDA technology. This allows a more accurate and robust determination of the membrane permeability of cells, including a relative quantification of these processes. The two assays are cost-effective in (routine) applications, but require access to a fluorescent flow cytometer and plate reader.

\section{Conflict of Interests}

The Authors declare that they have no competing interests in regard to this study. 


\section{Authors' Contributions}

Methodology: AN, LH, RE, DOM. Formal analysis: AN, LH, RE, DOM, MBS. Investigation: AN, LH, MBS. Writing, original draft preparation: MBS. Writing, review \& editing: MTZ, AE, MB, MBS. Supervision: MBS. Project administration: MBS. Funding acquisition: MTZ, AE, MB.

\section{References}

1 Escriba PV and Nicolson GK: Membrane structure and function: Relevance of lipid and protein structures in cellular physiology, pathology and therapy. Biochim Biophys Acta Biomembr 1838: 1449-1450, 2014. PMID: 24745792. DOI: 10.1016/ j.bbamem.2014.03.008

2 Yang NJ and Hinner MJ: Getting across the cell membrane: An overview for small molecules, peptides, and proteins. Methods Mol Biol 1266: 29-53, 2015. PMID: 25560066. DOI: 10.1007/ 978-1-4939-2272-7_3

3 Kanade S, Nataraj G, Ubale $M$ and Mehta P: Fluorescein diacetate vital staining for detecting viability of acid-fast bacilli in patients on antituberculosis treatment. Int J Mycobacteriol 5: 294-298, 2016. PMID: 27847013. DOI: 10.1016/j.ijmyco. 2016.06.003

4 Bätz FM, Klipper W, Korting HC, Henkler F, Landsiedel R, Luch A, von Fritschen U, Weindl $G$ and Schäfer-Korting M: Esterase activity in excised and reconstructed human skinbiotransformation of prednicarbate and the model dye fluorescein diacetate. Eur J Pharm Biopharm 84: 374-385, 2013. PMID: 23201050. DOI: 10.1016/j.ejpb.2012.11.008

5 Cao G, Zhang M, Miao J, Li W, Wang J, Lu D and Xia J: Effects of X-ray and carbon ion beam irradiation on membrane permeability and integrity in Saccharomyces cerevisiae cells. J Radiat Res 56: 294-304, 2015. PMID: 25599994. DOI: 10.1093/ $\mathrm{jrr} / \mathrm{rru} 114$

6 Armour AD, Powell HM and Boyce ST: Fluorescein diacetate for determination of cell viability in tissue-engineered skin. Tissue Eng Part C Methods 14: 89-96, 2008. PMID: 18454649. DOI: $10.1089 / \mathrm{tec} .2007 .0228$

7 Ambriz-Aviña V, Contreras-Garduño JA and Pedraza-Reyes M: Applications of flow cytometry to characterize bacterial physiological responses. Biomed Res Int 2014: 461941, 2014. PMID: 25276788. DOI: 10.1155/2014/461941

8 Clarke JM, Gillings MR, Altavilla N and Beattie AJ: Potential problems with fluorescein diacetate assays of cell viability when testing natural products for antimicrobial activity. J Microbiol Methods 46: 261-267, 2001. PMID: 11438191. DOI: doi.org/ $10.1016 / \mathrm{S} 0167-7012(01) 00285-8$
9 Karlsson H, Fryknäs M, Larsson R and Nygren P: Loss of cancer drug activity in colon cancer HCT-116 cells during spheroid formation in a new 3-D spheroid cell culture system. Exp Cell Res 318: 1577-1585, 2012. PMID: 22487097. DOI: 10.1016/j.yexcr.2012.03.026

10 Rinkevich FD, Margotta JW, Pittman JM, Danka RG, Tarver MR, Ottea JA and Healy KB: Genetics, synergists, and age affect insecticide sensitivity in the honey bee, Apis mellifera. PLoS One 10: e0139841.588, 2015. PMID: 26431171. DOI: 10.1371/journal.pone. 0139841

11 Schumacher TE, Eynard A and Chintala R: Rapid cost-effective analysis of microbial activity in soils using modified fluorescein diacetate method. Environ Sci Pollut Res In 22: 4759-4762, 2015. PMID: 25471725. DOI: 10.1007/s11356-014-3922-4

12 Bätz FM, Klipper W, Korting HC, Henkler F, Landsiedel R, Luch A, von Fritschen U, Weindl $\mathrm{G}$ and Schäfer-Korting M: Esterase activity in excised and reconstructed human skin biotransformation of prednicarbate and the model dye fluorescein diacetate. Eur J Pharm Biopharm 84: 374-385, 2013. PMID: 23201050. DOI: 10.1016/j.ejpb.2012.11.008

13 Johnson RM, Dahlgren L, Siegfried BD and Ellis MD: Acaricide, fungicide and drug interactions in honey bees (Apis mellifera). PLoS One 8(1): e54092, 2013. PMID: 23382869. DOI: 10.1371 /journal.pone .0054092

14 Marquez-Curtis LA, Sultani AB, McGann LE and Elliott JA: Beyond membrane integrity: Assessing the functionality of human umbilical vein endothelial cells after cryopreservation. Cryobiology 72: 183-190, 2016. PMID: 27182035. DOI: 10.1016/j.cryobiol.2016.05.005

15 Ahrend H, Kaul A, Ziegler S, Brandenburg LO, Zimmermann $\mathrm{U}$, Mustea A, Burchardt $\mathrm{M}$, Ziegler $\mathrm{P}$ and Stope $\mathrm{MB}$ : MicroRNA-1 and microRNA-21 individually regulate cellular growth of non-malignant and malignant renal cells. In Vivo 31: 625-630, 2017. PMID: 28652429. DOI: 10.21873/invivo.11103

16 Gelbrich N, Ahrend H, Kaul A, Brandenburg LO, Zimmermann U, Mustea A, Burchardt M, Gümbel D and Stope MB: Different cytokine and chemokine expression patterns in malignant compared to those in nonmalignant renal cells. Anal Cell Pathol 2017: 7190546, 2017. PMID: 28775934. DOI: 10.1155/ $2017 / 7190546$
Received July 4, 2019

Revised July 23, 2019

Accepted July 30, 2019 